OPEN ACCESS

Edited by:

Alemka Markotic,

University Hospital for Infectious Diseases "Dr Fran Mihaljevic", Croatia

Reviewed by: Jan Clement,

KU Leuven, Belgium William Marciel de Souza, University of São Paulo Ribeirão

Preto, Brazi

*Correspondence:

Chantal B. E. M. Reusken

Chantal.Reusken@rivm.nl

${ }^{\dagger}$ Present address:

Tabitha E. Hoornweg,

Faculty of Veterinary Medicine, Utrecht

University, Utrecht, Netherlands

Specialty section:

This article was submitted to

Virus and Host,

a section of the journal

Frontiers in Cellular and Infection Microbiology

Received: 06 July 2020 Accepted: 16 November 2020 Published: 22 December 2020

Citation:

Hoornweg TE, Zutt I, de Vries A, Maas M, Hoogerwerf MN, Avšič-

Županc T, Korva M, Reimerink JHJ and Reusken CBEM (2020)

Development of a Comparative European Orthohantavirus Microneutralization Assay With MultiSpecies Validation and Evaluation in a Human Diagnostic Cohort. Front. Cell. Infect. Microbiol. 10:580478.

doi: 10.3389/fcimb.2020.580478

\section{Development of a Comparative European Orthohantavirus Microneutralization Assay With Multi- Species Validation and Evaluation in a Human Diagnostic Cohort}

\author{
Tabitha E. Hoornweg ${ }^{1 \dagger}$, Ilse Zutt ${ }^{1}$, Ankje de Vries ${ }^{1}$, Miriam Maas ${ }^{1}$, \\ Marieke N. Hoogerwerf ${ }^{1}$, Tatjana Avšič-Županc ${ }^{2}$, Miša Korva ${ }^{2}$, Johan H. J. Reimerink ${ }^{1}$ \\ and Chantal B. E. M. Reusken ${ }^{1 *}$ \\ ${ }^{1}$ Centre for Infectious Disease Control, National Institute for Public Health and the Environment (RIVM), Bilthoven,
Netherlands, ${ }^{2}$ Institute of Microbiology and Immunology, Faculty of Medicine, University of Ljubljana, Ljubljana, Slovenia
}

Orthohantaviruses (family Hantaviridae, order Bunyavirales) can cause two serious syndromes in humans: hemorrhagic fever with renal syndrome (HFRS), associated with the Old World orthohantaviruses, and hantavirus cardiopulmonary syndrome (HCPS), associated with orthohantaviruses in the Americas. In Europe, four different orthohantaviruses (DOBV, PUUV, SEOV, and TULV) are associated with human disease. As disease severity and zoonotic source differ between orthohantavirus species, conclusive determination of the infecting species by either RT-PCR or comparative virus neutralization test (VNT) is of importance. Currently, the focus reduction neutralization test (FRNT) is considered the 'Gold Standard' for orthohantavirus VNTs, however this test is laborious and time-consuming. Consequently, more high-throughput alternatives are needed. In this study, we developed a comparative orthohantavirus microneutralization test (MNT) including all four human pathogenic orthohantavirus species circulating in Europe. The assay was validated using RT-PCR-confirmed rodent $(n=17)$ and human sera $(n=17)$, DOBVsuspected human sera $(n=3)$ and cohorts of orthohantavirus-negative rodent $(n=3)$ and human sera ( $n=85)$. 16/17 RT-PCR-confirmed rodent sera and 18/20 of the RT-PCRconfirmed and DOBV-suspected human sera were serotyped successfully, while for the remaining rodent $(n=1)$ and human sera $(n=2)$ no neutralizing titers could be detected. All negative control sera tested negative in the MNT. The assay was subsequently evaluated using a clinical cohort of 50 orthohantavirus patients. Orthohantavirus infection was confirmed in all 50 patients, and 47/50 (94\%) sera were serotyped successfully, confirming PUUV as the major cause of orthohantavirus infections in Netherlands. Notably, two previously unrecognized SEOV cases from 2013 were diagnosed using the MNT, underlining the added value of the MNT in a diagnostic setting. In conclusion, we demonstrate the successful development and clinical implementation of a comparative 
European orthohantavirus MNT to determine the infecting virus species in European HFRS patients. Identification of the causative species is needed for an adequate Public Health response and can support individual patient care. For many labs, the implementation of orthohantavirus neutralization tests has not been a straightforward procedure. This issue will be addressed by the rollout of the comparative MNT to multiple European laboratories to support patient diagnostics, surveillance and Public Health responses.

Keywords: orthohantaviruses, Dobrava virus, Puumala virus, Seoul virus, Tula virus, virus neutralization test, microneutralization test, serotyping

\section{INTRODUCTION}

Orthohantaviruses are enveloped negative stranded RNA viruses with a tripartite genome that belong to the family of Hantaviridae, order Bunyavirales (Reuter and Kruger, 2018). Each orthohantavirus species is carried by one or few closely related species of reservoir host(s), either rodents, shrews, moles or bats (Vapalahti et al., 2003; Reusken and Heyman, 2013). As a consequence the distribution of orthohantaviruses is strictly dependent on the dispersal of their reservoir host. Although orthohantaviruses typically cause chronic infections without overt disease in their reservoir host (Vaheri et al., 2013), in humans they may cause serious illness. While old world orthohantaviruses are mainly associated with hemorrhagic fever with renal syndrome (HFRS), new world orthohantaviruses are primarily associated with hantavirus cardiopulmonary syndrome (HCPS). However, it is increasingly being recognized that considerable clinical overlap exists between both syndromes (Clement et al., 2014).

Four different orthohantavirus species are associated with human disease in Europe: Dobrava/Belgrade virus (DOBV) carried by three closely related species of the genus Apodemus; Puumala virus (PUUV) carried by the bank vole (Myodes glareolus); Seoul virus (SEOV) carried by different species of rats (Rattus spp.); and Tula virus (TULV) carried by the common vole (Microtus arvalis) (Reusken and Heyman, 2013). Additionally, while Hantaan virus (HTNV), carried by the striped field mouse (A. agrarius), is generally believed to be only present in Asia, some studies suggested that it may also be present in eastern parts of Europe (Clement et al., 1997).

Of the orthohantaviruses with confirmed presence in Europe, DOBV is associated with the most severe form of HFRS with case fatality ratio's (CFR) up to $12 \%$, depending on the genotype (Klempa et al., 2013). SEOV is associated with a mild-moderate form of HFRS, whereas PUUV causes a relatively mild form of HFRS, also known as Nephropathia Epidemica (NE) (Vapalahti et al., 2003; Kruger et al., 2011; Reusken and Heyman, 2013). To date, TULV has only been associated with a low number of human infections, which were either considered asymptomatic or in which a mild form of HFRS was observed (Vapalahti et al., 1996; Schultze et al., 2002; Clement et al., 2003; Klempa et al., 2003; Zelena et al., 2013; Reynes et al., 2015).

In Netherlands, PUUV, SEOV and TULV all circulate in their respective reservoir hosts (Reusken et al., 2008; Verner-Carlsson et al., 2015; de Vries et al., 2016; Maas et al., 2017; Swanink et al., 2018). Each year approximately 25-30 human orthohantavirus infections are reported, although this is likely an underestimation (Goeijenbier et al., 2014). Due to the high genetic variability within the orthohantavirus species and the notion that in many cases no viral RNA can be detected in the first clinical samples sent to diagnostic laboratories, laboratory diagnostics are primarily based on serology (Vapalahti et al., 2003). The pathogenic orthohantaviruses present in Europe can be subdivided into two different serogroups (Vaheri et al., 2008); PUUV and TULV belong to the serogroup of Arvicolinae-borne orthohantaviruses, while DOBV and SEOV (together with HNTV) are part of the serogroup of Murinae-borne orthohantaviruses. Unfortunately, however, as high levels of cross-reactive antibodies are observed within but also between the different serogroups (albeit to a lesser extent), the infecting virus species cannot be determined conclusively using routine serological assays (Vapalahti et al., 2003). As a consequence, in Netherlands, orthohantavirus infections are routinely attributed to PUUV which is assumed to be the most widespread orthohantavirus in the country (Sane et al., 2014). Yet, as disease severity and the zoonotic source differ between orthohantavirus species, conclusive determination of the infecting species is of importance for surveillance, source attribution, risk management and monitoring of control measures.

Species determination of the infecting orthohantavirus can only be done by RT-PCR or comparative virus neutralization assays (VNT). To date, multiple variations of orthohantavirus neutralization assays have been described (Tanishita et al., 1984; Lee et al., 1985; Takenaka et al., 1985; Niklasson et al., 1991; Horling et al., 1992; Chu et al., 1995; Heider et al., 2001; Maes et al., 2009; Padua et al., 2015; Li et al., 2017), however, the focus reduction neutralization test (FRNT) is generally viewed as the 'Gold Standard'. Unfortunately, the orthohantavirus FRNT is rather laborious and time consuming and therefore not very suitable for assaying a large number of samples (Horling et al., 1992; Li et al., 2017). Recently, Li et al. (2017) showed that the more high-throughput microneutralization test (MNT) was a good alternative to the FRNT when serotyping human infections with the closely related HTNV and SEOV (Li et al., 2017). In this study, we describe the development, validation and evaluation of a comparative orthohantavirus MNT including all four human pathogenic orthohantavirus species circulating in Europe. 


\section{MATERIALS AND METHODS}

\section{Cells, Viruses, and Antibodies}

Green monkey kidney Vero-E6 cells (ATCC C1008 CRL1586) were maintained in Dulbecco's Modified Eagle's MEM (DMEM, Gibco) supplemented with 5\% heat-inactivated fetal bovine serum (FBS; Biowest, Nuaillé, France), $100 \mathrm{U} / \mathrm{ml}$ penicillin and $100 \mathrm{U} / \mathrm{ml}$ streptomycin (Biowest, Nuaillé, France). Cells were cultured at $37^{\circ} \mathrm{C}$ under $5 \% \mathrm{CO}$.

DOBV strain SK/Aa (catalog number: 008v-EVA1470), PUUV strain Sotkamo (catalog number: 008v-EVA1472), SEOV strain 80-39 (catalog number: 008v-EVA1473), and TULV strain Moravia (no longer available from EVAg) were all acquired via the European Virus Archive (EVAg). Stocks were created by passaging twice on Vero-E6 cells using a low multiplicities of infection (MOI 0.001-0.1), and harvesting 714 days post infection.

\section{Virus Titration}

Titers of the virus stocks were determined by use of a $50 \%$ tissue culture infectious dose $\left(\mathrm{TCID}_{50}\right)$ assay adapted for indirect detection of virus-infected cells. Briefly, tenfold viral dilutions were inoculated on Vero-E6 cells and incubated for 7 (DOBV, SEOV and TULV) or 12 days (PUUV) at $37^{\circ} \mathrm{C}$, which corresponded to peak titers during virus culture (data not shown). Subsequently, cells were fixed using $4 \% \mathrm{PFA}$ and permeabilized using $0.02 \%$ Triton X-100 in PBS. Subsequently, cells were washed with PBS and stained using mouse anti-hanta nucleoprotein (1:500; abcam, Cambridge, United Kingdom) (for PUUV, SEOV and TULV) or mouse anti-hantavirus Dobrava strain nucleoprotein (1:1,000; IBTsystems, Binzwangen, Germany) (for DOBV) as primary antibodies and goat antimouse-AlexaFluor488 (1:200; Abcam, Cambridge, United Kingdom) as a secondary antibody. Positive wells were visualized using a Leica DMIL LED fluorescence microscope and scored by eye. $\mathrm{TCID}_{50}$ titers were calculated using the Spearman and Kärber algorithm (Hierholzer and Killington, 1996).

\section{Microneutralization Test}

The orthohantavirus neutralization test was designed as a microneutralization tests (MNT) as previously described for the Influenza A virus and the orthohantaviruses HTNV and SEOV (Klimov et al., 2012; Li et al., 2017) with an adaptation for a fluorescence-based read-out. Briefly, two-fold serial dilutions (starting dilution 1:40) of heat-inactivated sera $\left(30 \mathrm{~min}\right.$ at $\left.56^{\circ} \mathrm{C}\right)$ were mixed with $100 \mathrm{TCID}_{50}$ of the appropriate virus species and incubated for $2 \mathrm{~h}$ at $37^{\circ} \mathrm{C}$. Subsequently, $100 \mu \mathrm{l}$ virus-antibody complexes were transferred to a 96 -wells plate containing a $90 \%$ confluent Vero-E6 monolayer. $1.5-3 \mathrm{~h}$ post infection, an additional $100 \mu \mathrm{l}$ Vero-E6 medium supplemented with $25 \mathrm{mM}$ HEPES was added to each well. Plates were incubated for 7 (DOBV, SEOV, TULV) or 12 (PUUV) days at $37^{\circ} \mathrm{C}$, before cells were fixed, permeabilized and stained as described above. Virus-positive cells were visualized using a Leica DMIL LED fluorescence microscope. The neutralization titer was determined by scoring the wells in which infection was inhibited $\geq 95 \%$ compared to the positive control $\left(\mathrm{MNT}_{95}\right)$.

\section{Serum Panels for Validation of the Orthohantavirus MNT}

For validation of the orthohantavirus MNT, a panel of 17 rodent sera representing the four different human pathogenic European orthohantavirus species and their associated reservoir was used, of which all specifications are included in Table 1. The rodent sera were both RT-PCR and IgG positive using routine serology for $\operatorname{DOBV}(\mathrm{n}=4)$, PUUV $(\mathrm{n}=5), \operatorname{SEOV}(\mathrm{n}=5)$, or TULV $(\mathrm{n}=3)$. The sera positive for DOBV, PUUV, and TULV originated from wild rodents caught for surveillance purposes in either Netherlands or Slovenia. The SEOV positive sera came from a SEOV-positive feeder rat colony associated with a human SEOV infection in Netherlands (Swanink et al., 2018)). Additionally, a small panel of wild caught $R$. norvegicus sera $(\mathrm{n}=3)$ that previously tested negative in both RT-PCR and the orthohantavirus FRNT in Uppsala, Sweden (Maas et al., 2018) was included as negative controls. All animal handling procedures were approved by the Dutch (DEC project numbers 200900164 and 201200208) and Slovenian Animal Ethics Committee (Administration for Food Safety, Veterinary Sector and Plant Protection: U34401-15/2018/8).

For the validation of the MNT with human sera, a serum panel from 17 patients with a RT-qPCR confirmed orthohantavirus infection diagnosed in the period of 20112017, was used. Eleven sera (9x PUUV-positive, 2x SEOVpositive) originated from Dutch patients and were diagnosed using the genotype-specific RT-qPCRs described by (Kramski et al., 2007), while six sera (5x DOBV-positive, 1x PUUVpositive (Korva et al., 2013b)) originated from Slovenian patients and were diagnosed with RT-qPCRs previously described by (Korva et al., 2009). For 11 patients the acute phase RT-qPCR-positive sample was directly tested in the MNT, while for six patients $(3 \times$ DOBV-positive, $2 \times$ PUUV-positive, $1 \times$ SEOV-positive) a subsequent paired serum sample to the RTqPCR-positive serum sample was tested in the MNT (paired sera are indicated by a ${ }^{*}$ in Table 4). Of the paired sera, all three sera from the DOBV-positive patients were late convalescent, while the sera from the PUUV- $(2 \times)$ and SEOV-positive $(1 \times)$ patients were drawn less than one month after the onset of symptoms. All patients consented that the remainder of their sera could be used for research purposes.

In addition, a small serum panel from DOBV-suspected human patients $(\mathrm{n}=3)$ kindly provided by Sabine Lederer from Euroimmun (Lubeck, Germany) was tested in the orthohantavirus MNT. All samples showed the highest IgG titers against DOBV in the Euroimmun mosaic immunofluorescent assay (IFA) (Lederer et al., 2013), with a varying degree of cross-reactivity toward the other (primarily Murinae-borne) orthohantaviruses. Two of three sera were known to be convalescent (both samples were taken more than one year after onset of symptoms), while for the third serum the status was unknown. None of these sera were confirmed by RT-PCR.

Finally, 85 randomly selected sera collected from healthy Dutch blood donors in 2016 were included as negative controls. 
TABLE 1 | Specifics of the rodent serum panel used for validation of the orthohantavirus microneutralization test (MNT).

\begin{tabular}{|c|c|c|c|c|c|c|c|c|}
\hline \multirow[t]{2}{*}{ Serum \# } & \multirow[t]{2}{*}{ Host } & \multirow[t]{2}{*}{ Country of Origin } & \multicolumn{3}{|c|}{ Serology } & \multicolumn{3}{|c|}{ RT-(q)PCR } \\
\hline & & & $+/-$ & Test & Value & $+/-$ & Segment & Species \\
\hline PUUV 1 & Myodes Glareolus & Netherlands & + & PUUV-IgG immunochromatography fast test & 185 & + & partial L & PUUV \\
\hline PUUV 2 & Myodes Glareolus & Netherlands & + & PUUV-lgG immunochromatography fast test & 168 & + & partial L & PUUV \\
\hline PUUV 4 & Myodes Glareolus & Netherlands & + & PUUV-IgG immunochromatography fast test & 129 & + & partial L & PUUV \\
\hline PUUV 6 & Myodes Glareolus & Slovenia & + & IFT PUUV/IFT DOBV & $+++/++$ & + & partial S + partial L & PUUV \\
\hline PUUV 7 & Myodes Glareolus & Slovenia & + & IFT PUUV/IFT DOBV & $++/+/-$ & + & partial S + partial L & PUUV \\
\hline TULV 1 & Microtus Arvalis & Netherlands & + & PUUV-lgG immunochromatography fast test & 12 & + & partial S & TULV \\
\hline TULV 2 & Microtus Arvalis & Netherlands & + & PUUV-IgG immunochromatography fast test & 13 & + & partial S & TULV \\
\hline TULV 3 & Microtus Arvalis & Netherlands & + & PUUV-IgG immunochromatography fast test & 10 & + & partial S & TULV \\
\hline rDOBV 1 & Apodemus flavicollis & Slovenia & + & IFT PUUV/IFT DOBV & $-/+$ & + & partial S + partial L & DOBV \\
\hline rDOBV 2 & Apodemus flavicollis & Slovenia & + & IFT PUUV/IFT DOBV & $+/++$ & + & partial S + partial L & DOBV \\
\hline rDOBV 3 & Apodemus flavicollis & Slovenia & + & IFT PUUV/IFT DOBV & $++/+++$ & + & partial S + partial L & DOBV \\
\hline rDOBV 4 & Apodemus agrarius & Slovenia & + & IFT PUUV/IFT DOBV & $+/+$ & + & partial $S+$ partial L & DOBV-Kurkino \\
\hline SEOV 1 & Rattus Norvegicus & Netherlands & + & adapted Hantavirus Dobrava/Hantaan IgG Elisa & 2.567 & + & partial S & HTNV/SEOV \\
\hline SEOV 2 & Rattus Norvegicus & Netherlands & + & adapted Hantavirus Dobrava/Hantaan IgG Elisa & 2.48 & + & partial S & HTNV/SEOV \\
\hline SEOV 3 & Rattus Norvegicus & Netherlands & + & adapted Hantavirus Dobrava/Hantaan IgG Elisa & 2.385 & + & partial S & HTNV/SEOV \\
\hline SEOV 4 & Rattus Norvegicus & Netherlands & + & adapted Hantavirus Dobrava/Hantaan IgG Elisa & 2.276 & + & partial S & HTNV/SEOV \\
\hline SEOV 5 & Rattus Norvegicus & Netherlands & + & adapted Hantavirus Dobrava/Hantaan IgG Elisa & 2.121 & + & partial S & HTNV/SEOV \\
\hline
\end{tabular}

The Puumala virus (PUUV)-IgG immunochromatography fast test, the PUUV and different orthohantaviruses (DOBV) IFT, and the adapted Hantavirus Dobrava/Hantaan IgG ELISA have previously been described in (de Vries et al., 2016), (Korva et al., 2013a) and (Swanink et al., 2018), respectively. Reported values either represent the quantified antibody levels as measured by the ReaScan Reader (PUUV-IgG immunochromatography fast test) or OD (adapted Hantavirus Dobrava/Hantaan IgG ELISA). For the IFT a semi-quantitative read-out was used, in which samples were scored -, +/-, +, ++, or +++ based on staining pattern and brightness. RT-(q)PCR protocols were previously described by (Razzauti et al., 2009) (PUUV partial L), (Korva et al., 2013a) (PUUV and DOBV partial S and partial L), and (Kramski et al., 2007) (TULV and HTNV/SEOV partial S). A selection of samples diagnosed by the PUUV partial $L$ and the HTNV/SEOV partial S RT-qPCR protocols were further confirmed by Sanger sequencing.

\section{Diagnostic Cohort}

Sera of 50 Dutch patients clinically suspected of an orthohantavirus infection, collected in the period 2013-2017, and diagnosed with an orthohantavirus infection based on routine serology were selected for testing in the orthohantavirus MNT. Initial routine orthohantavirus diagnostics were performed using the Euroimmun mosaic IFA (Lederer et al., 2013), in which samples are simultaneously tested against six clinically important orthohantaviruses (DOBV, HTNV, PUUV, Saaremaa virus (SAAV), SEOV and Sin Nombre Virus (SNV)), according to manufacturer's instructions (Euroimmun, Lubeck, Germany). Sera were considered positive for orthohantavirus infection when IgM and/or IgG titers were $\geq 128$ for at least one of the orthohantaviruses tested (Lederer et al., 2013; Geurts van Kessel et al., 2016). All serum samples were tested negative for the presence of DOBV, PUUV, SEOV/HTNV, and TULV RNA using the serotype-specific RTqPCRs described by (Kramski et al., 2007). Further specifications of the cohort are listed in Table 2. For all samples, the orthohantavirus MNT was performed in parallel for all four orthohantaviruses species. When paired sera were available, the serum sample of the latest collection date was tested in the MNT. All patients consented that the remainder of their sera could be used for research purposes.

\section{RESULTS}

\section{Development of a Fluorescent-Based "European" Orthohantavirus Microneutralization Assay}

The aim of this work was to set up a MNT including all human pathogenic orthohantaviruses circulating in Europe, namely
DOBV, PUUV, SEOV, and TULV. Initially, we designed the European orthohantavirus MNT according to the approach of (Li et al., 2017), in which infection is quantified by measuring the enzymatic activity of horseradish peroxidase (HRP). However, as for both PUUV and TULV only relatively low OD values could be measured under positive control conditions (infection with $100 \mathrm{TCID}_{50}$ ), it was not possible to determine levels of neutralization of PUUV and TULV infection robustly using this approach (data not shown).

Consequently, the MNT was designed with a fluorescencebased read-out, in which the $\mathrm{MNT}_{95}$ titer, corresponding to the highest antibody dilution that inhibited infection $\geq 95 \%$, was determined by eye. As shown in Supplementary Figure 1, virusinfected cells could be clearly discerned for all orthohantaviruses used in this study. The $\mathrm{MNT}_{95}$ titer was preferred over an endpoint neutralization titer (complete inhibition of infection), as at times small breakthrough infections could be observed that prevented the determination of an end-point neutralization titer, even though clear neutralization was observed.

\section{Validation: RT-PCR-Confirmed Rodent Sera}

For initial validation of the MNT assay, a panel of sera $(n=17)$ from multiple rodent species, previously determined to be serologically ( $\operatorname{IgG}$ ) and RT-PCR positive for either DOBV, PUUV, SEOV or TULV, was used. Table 3 shows the $\mathrm{MNT}_{95}$ titers of the rodent serum validation panel. Neutralization titers of $\geq 40$ were considered positive (Kallio-Kokko et al., 2006; Hofmann et al., 2014). Additionally, when positive $\mathrm{MNT}_{95}$-titers against multiple orthohantavirus species were found, a titer difference of $\geq 4$ in the comparative tests was required for definitive serotyping (Nichol et al., 2005). 
TABLE 2 | General characteristics of the diagnostic cohort of clinically suspected human orthohantavirus cases diagnosed with anorthohantavrus infection based on routine serology.

\begin{tabular}{|c|c|}
\hline Variable & $\begin{array}{l}\text { Orthohantaviru } \\
\text { infections } \\
(n=50)\end{array}$ \\
\hline \multicolumn{2}{|l|}{ Gender - No. (\%) } \\
\hline Male & $35(70.0)$ \\
\hline Female & $15(30.0)$ \\
\hline \multicolumn{2}{|l|}{ Age - years } \\
\hline Median & 46 \\
\hline Range & $15-77$ \\
\hline \multicolumn{2}{|l|}{ Age group - No. (\%) } \\
\hline$<20$ years & $4(8.0)$ \\
\hline $21-40$ years & $17(34.0)$ \\
\hline $41-60$ years & $19(38.0)$ \\
\hline$>61$ years & $10(20.0)$ \\
\hline \multicolumn{2}{|c|}{ Serogroup with highest reactivity in IFA - No. (\%) } \\
\hline PUUV/SNV & $37(74.0)$ \\
\hline DOBV/HTNV/SAAV/SEOV & $4(8.0)$ \\
\hline No difference* & $9(18.0)$ \\
\hline \multicolumn{2}{|c|}{$\begin{array}{l}\text { Serum collection (days post onset of infection) - No. } \\
(\%)\end{array}$} \\
\hline$<30$ & $16(32.0)$ \\
\hline$>30$ & $6(12.0)$ \\
\hline Unknown & $28(56.0)$ \\
\hline \multicolumn{2}{|l|}{ Travel History - No. (\%) } \\
\hline Yes & $5(10.0)$ \\
\hline No & $1(2.0)$ \\
\hline Unknown & $44(88.0)$ \\
\hline
\end{tabular}

$A$ * indicates that at the highest dilution tested, samples were IgM and IgG positive for both serogroups.

Close to all rodent sera $(16 / 17)$ had distinct neutralizing titers toward the infecting virus species and could be serotyped as expected (Table 3). For one of the rodent sera positive for DOBV (rDOBV4, derived from A. agrarius), no neutralization was observed. As this serum was only weakly positive in the IFT, it is possible that the serum was taken early after infection of the respective rodent and neutralizing antibodies were not yet present at the time of sampling.

For most sera $(n=11 / 17)$, some degree of cross-neutralization toward the non-infecting other orthohantaviruses was observed, which was most prominent toward the orthohantaviruses from the same serogroup (PUUV $\leftrightarrow$ TULV and SEOV $\rightarrow$ DOBV) (Vaheri et al., 2008). Cross-neutralization toward viruses belonging to the other serogroup (PUUV/TULV $\leftrightarrow$ DOBV/SEOV) was generally low (maximal cross-reactive titer observed: 1280 for SEOV, 80 for PUUV (SEOV3)), and only observed for the SEOV-positive sera. Nevertheless, a $\geq 4$-fold difference in neutralizing titers was observed for all MNT positive sera, and the infecting virus could be confidently serotyped for each serum.

As a negative control, wild-caught $R$. norvegicus sera that were found to be positive in a genus-wide orthohantavirus ELISA but were previously determined to be negative in both RT-PCR and an orthohantavirus FRNT (Maas et al., 2018), were tested in the MNT. In line with the FRNT results, all sera were negative in the $\mathrm{MNT}_{95}$ (data not shown).

\section{Validation: RT-PCR-Confirmed Human Patient Sera}

Next, we aimed to validate the orthohantavirus MNT using sera of RT-qPCR-confirmed human orthohantavirus cases ( $n=17 ; 11$ acute sera and six paired serum samples of a later sampling date). For this purpose, we used sera from 11 Dutch and six Slovenian patients with a RT-qPCR-confirmed orthohantavirus infection diagnosed in the period from 2011 to 2017. Of these patients, 10 were RT-qPCR positive for PUUV (9 Dutch patients and 1 Slovenian patient), two were positive for SEOV (both Dutch patients) and five were positive for DOBV (all Slovenian

TABLE 3 | MNT 95 titers of rodent sera used for orthohantavirus microneutralization test (MNT) validation.

\begin{tabular}{|c|c|c|c|c|c|c|c|}
\hline \multirow[t]{3}{*}{ Serum \# } & \multirow[t]{3}{*}{ Host } & \multicolumn{6}{|c|}{ Orthohantavirus $\mathrm{MNT}_{95}$} \\
\hline & & \multicolumn{4}{|c|}{$95 \%$ neutralization titer } & \multirow[t]{2}{*}{ Minimal Fold change } & \multirow[t]{2}{*}{ Conclusion } \\
\hline & & PUUV & TULV & DOBV & SEOV & & \\
\hline PUUV 1 & Myodes glareolus & $\geq 5,120$ & 160 & $<40$ & $<40$ & $\geq 32$ & PUUV \\
\hline PUUV 2 & Myodes glareolus & $\geq 5,120$ & 160 & $<40$ & $<40$ & $\geq 32$ & PUUV \\
\hline PUUV 4 & Myodes glareolus & $\geq 5,120$ & 80 & $<40$ & $<40$ & $\geq 64$ & PUUV \\
\hline PUUV 6 & Myodes glareolus & $\geq 5,120$ & $<40$ & $<40$ & $<40$ & $>128$ & PUUV \\
\hline PUUV 7 & Myodes glareolus & $\geq 5,120$ & $<40$ & $<40$ & $<40$ & $>128$ & PUUV \\
\hline TULV 1 & Microtus arvalis & 80 & 2,560 & $<40$ & $<40$ & 32 & TULV \\
\hline TULV 2 & Microtus arvalis & 80 & 2,560 & $<40$ & $<40$ & 32 & TULV \\
\hline TULV 3 & Microtus arvalis & 640 & $\geq 5,120$ & $<40$ & $<40$ & $\geq 8$ & TULV \\
\hline rDOBV1 & Apodemus flavicollis & $<40$ & $<40$ & 320 & $<40$ & $\geq 16$ & DOBV \\
\hline rDOBV2 & Apodemus flavicollis & $<40$ & $<40$ & 80 & $<40$ & $\geq 4$ & DOBV \\
\hline rDOBV3 & Apodemus flavicollis & $<40$ & $<40$ & 640 & $<40$ & $\geq 32$ & DOBV \\
\hline rDOBV4 & Apodemus agrarius & $<40$ & $<40$ & $<40$ & $<40$ & - & Negative \\
\hline SEOV 1 & Rattus norvegicus & 80 & $<40$ & 1,280 & $\geq 5,120$ & $\geq 4$ & SEOV \\
\hline SEOV 2 & Rattus norvegicus & 40 & $<40$ & $<40$ & $\geq 5,120$ & $\geq 128$ & SEOV \\
\hline SEOV 3 & Rattus norvegicus & 80 & $<40$ & 320 & 1,280 & 4 & SEOV \\
\hline SEOV 4 & Rattus norvegicus & 80 & $<40$ & 320 & $\geq 5,120$ & $\geq 16$ & SEOV \\
\hline SEOV 5 & Rattus norvegicus & $<40$ & $<40$ & 640 & $\geq 5,120$ & $\geq 8$ & SEOV \\
\hline
\end{tabular}

Neutralization titers of $\geq 40$ were considered positive and, when neutralizing titers against multiple orthohantaviruses were detected, a titer difference of $\geq 4$-fold was required for definitive serotyping. The highest positive titer is highlighted in gray. 
patients). As human TULV infections are rare, no sera from RTqPCR confirmed TULV patients were available.

Neutralizing titers for the RT-qPCR-confirmed patient sera are shown in Table 4. All PUUV $(n=10)$ and DOBV $(n=5)$ cases could be serotyped correctly using the orthohantavirus MNT. For SEOV, however, both cases were negative in the MNT. One of these sera (17-07b) did show visible neutralization at the lowest antibody dilutions (i.e. 40 and 80); however the extent of neutralization did not reach 95\%. Although this serum sample was a paired sample to a previous RT-qPCR-positive serum sample and showed clear IgM and IgG titers in the diagnostic IFA, the exact period between onset of symptoms and serum sampling were unknown. Consequently, it remains unknown whether the sample tested was either acute or (early) convalescent. For the other serum (17-08), no neutralization was observed. This serum was an acute phase serum that was still positive for SEOV in the RT-qPCR. Likely, this serum was taken too early after infection for neutralizing antibodies to develop.

\section{Validation: Sera From Human Patients With a Suspected DOBV Infection}

In addition to the RT-PCR-confirmed rodent and human sera, a small serum panel $(n=3$, including two convalescent sera) from patients with a suspected DOBV infection was tested in the MNT. As expected, sera of all three patients were serotyped as DOBV infections (Table 5).

\section{Validation: Dutch Blood Bank Cohort}

As the incidence of orthohantavirus infections in Netherlands is relatively low (Sane et al., 2014), 85 randomly selected serum samples from healthy Dutch blood donors were included as negative controls during validation of the orthohantavirus MNT.
As expected, all sera tested negative (data not shown), implying that neutralization is not caused by cytotoxic or virocidal components in serum, but is orthohantavirus specific.

\section{Evaluation: Diagnostic Cohort of Clinically Suspected Human Orthohantavirus Cases Diagnosed With an Orthohantavirus Infection Based on Routine Serology}

To evaluate the MNT in a clinical diagnostic setting, a cohort consisting of 50 Dutch patients diagnosed with an orthohantavirus infection based on routine serology was tested in the comparative MNT. All sera (100\%) showed neutralizing titers against at least one of the orthohantaviruses, and could thus be confirmed as orthohantavirus infections based on Gold Standard serology (Table 6). In addition, 47/50 sera (94\%) could be serotyped conclusively using the MNT. The vast majority (44) were serotyped as PUUV infections, whereas three sera were found to be positive for SEOV. Without exclusion, the orthohantavirus that caused the infection as identified by MNT-based serotyping was part of the serogroup to which the highest titers were found during routine diagnostics using the mosaic IFA.

Of the sera that could not be serotyped, two sera (15-03 and 16-12) showed a neutralizing titer for both PUUV and TULV with only a two-fold difference, and were thus considered inconclusive. A third inconclusive serum (17-09b) showed a rather atypical neutralization profile of cross-neutralization between the different serogroups (both TULV and SEOV were positive with $\mathrm{MNT}_{95}$ titers of 80 and 40, respectively) while no cross-neutralization within the serogroups was observed (both DOBV and PUUV were negative). Most likely this patient was infected with SEOV, as the patient owned SEOV-positive rats.

TABLE 4 | MNT 95 titers of patient sera with a RT-PCR-confirmed orthohantavirus infection.

\begin{tabular}{|c|c|c|c|c|c|c|c|c|c|}
\hline \multirow[t]{3}{*}{ Patient ID } & \multirow[t]{3}{*}{ Country of Origin } & \multicolumn{2}{|c|}{ RT-qPCR } & \multicolumn{6}{|c|}{ Orthohantavirus $\mathrm{MNT}_{95}$} \\
\hline & & \multirow[b]{2}{*}{$+/-$} & \multirow[b]{2}{*}{ Species } & \multicolumn{4}{|c|}{$95 \%$ neutralization titer } & \multirow[t]{2}{*}{ Minimal Fold change } & \multirow[t]{2}{*}{ Conclusior } \\
\hline & & & & PUUV & TULV & DOBV & SEOV & & \\
\hline $14-05$ & Netherlands & + & PUUV & 1,280 & $<40$ & $<40$ & $<40$ & $\geq 64$ & PUUV \\
\hline $14-08$ & Netherlands & + & PUUV & 640 & 40 & $<40$ & $<40$ & 16 & PUUV \\
\hline $16-07$ & Netherlands & + & PUUV & 320 & 40 & $<40$ & $<40$ & 8 & PUUV \\
\hline $17-02$ & Netherlands & + & PUUV & 320 & $<40$ & $<40$ & $<40$ & $\geq 16$ & PUUV \\
\hline $17-06 b$ & Netherlands & $+^{*}$ & PUUV & 1,280 & $<40$ & $<40$ & $<40$ & $\geq 64$ & PUUV \\
\hline $17-13$ & Netherlands & + & PUUV & 640 & 80 & $<40$ & $<40$ & 8 & PUUV \\
\hline $17-14$ & Netherlands & + & PUUV & 640 & 160 & $<40$ & $<40$ & 4 & PUUV \\
\hline $17-23$ & Netherlands & + & PUUV & 320 & $<40$ & $<40$ & $<40$ & $\geq 16$ & PUUV \\
\hline $17-25 b$ & Netherlands & $+^{*}$ & PUUV & $\geq 5,120$ & 640 & $<40$ & $<40$ & $\geq 8$ & PUUV \\
\hline 580 & Slovenia & + & PUUV & 320 & $<40$ & $<40$ & $<40$ & $\geq 16$ & PUUV \\
\hline $17-07 b$ & Netherlands & $+^{*}$ & SEOV & $<40$ & $<40$ & $<40$ & $<40$ & - & Negative \\
\hline $17-08$ & Netherlands & + & SEOV & $<40$ & $<40$ & $<40$ & $<40$ & - & Negative \\
\hline 306 & Slovenia & $+^{*}$ & DOBV & $<40$ & $<40$ & 80 & $<40$ & $\geq 4$ & DOBV \\
\hline 465 & Slovenia & $+^{\star}$ & DOBV & $<40$ & $<40$ & 40 & $<40$ & $\geq 2$ & DOBV \\
\hline 625 & Slovenia & + & DOBV & $<40$ & $<40$ & 40 & $<40$ & $\geq 2$ & DOBV \\
\hline 632 & Slovenia & $+^{*}$ & DOBV & $<40$ & $<40$ & 160 & $<40$ & $\geq 8$ & DOBV \\
\hline 548 & Slovenia & + & DOBV-Kurkino & $<40$ & $<40$ & 160 & $<40$ & $\geq 8$ & DOBV \\
\hline
\end{tabular}

Neutralization titers of $\geq 40$ were considered positive and, when neutralizing titers are measured against multiple orthohantaviruses, a titer difference of $\geq 4$-fold was required for definitive serotyping. The highest positive titer is highlighted in gray. For the samples marker with *, a paired sample to a previous RT-PCR-positive serum sample was tested in the microneutralization test (MNT). 
TABLE 5 | MNT 95 titers for different orthohantaviruses (DOBV)-suspected patient sera.

\begin{tabular}{|c|c|c|c|c|c|c|}
\hline \multirow[t]{3}{*}{ Serum \# } & \multicolumn{6}{|c|}{ Orthohantavirus $\mathrm{MNT}_{95}$} \\
\hline & \multicolumn{4}{|c|}{$95 \%$ neutralization titer } & \multirow[t]{2}{*}{ Minimal Fold change } & \multirow[t]{2}{*}{ Conclusion } \\
\hline & PUUV & TULV & DOBV & SEOV & & \\
\hline DOBV suspected 1 & $<40$ & $<40$ & 320 & $<40$ & $\geq 16$ & DOBV \\
\hline DOBV suspected 2 & $<40$ & $<40$ & 40 & $<40$ & $\geq 2$ & DOBV \\
\hline DOBV suspected 3 & 40 & 40 & 640 & 80 & 8 & DOBV \\
\hline
\end{tabular}

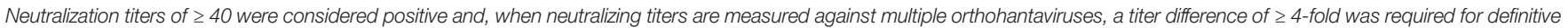
serotyping. The highest positive titer is highlighted in gray.

\section{DISCUSSION}

Here, we describe the successful development and implementation of a MNT including all four European human pathogenic orthohantavirus species. In a clinical cohort of orthohantavirus patients, all patients could be confirmed, with conclusive identification of the causative orthohantavirus species for $94 \%$ of the patients. Our data shows that the orthohantavirus MNT represents a more high-throughput alternative to the orthohantavirus PRNT (the Gold Standard in orthohantavirus VNTs) for serotyping orthohantavirus infections. This is in line with two previous studies (Horling et al., 1992; Li et al., 2017) that showed that the MNT, albeit in slightly different set-ups, was as a viable alternative to the PRNT for the detection of PUUV neutralizing antibodies (Horling et al., 1992) and serotyping human HTNV and SEOV infections (Li et al., 2017).

The MNT was validated using 17 rodent (Table 3) and 17 human sera of RT-PCR-confirmed infections (Table 4), with the addition of three human sera of suspected DOBV infections (Table 5). For the rodent sera, 16/17 sera were serotyped in line with RT-PCR outcomes, while one DOBV-Kurkino positive $A$. agrarius serum remained negative in the MNT (Table 3 rDOBV4). As this serum only showed low IgG levels in the IFT, it is possible that the serum was taken too early after infection for neutralizing antibodies to form.

All PUUV- and DOBV-confirmed/suspected human sera were serotyped as expected. However, both RT-qPCRconfirmed SEOV sera remained negative in the MNT at $95 \%$ cut-off (Table 4 - 17-07b and 17-08). One of the serum samples tested was from a patient that was still viremic (17-08). For the second patient, a paired serum to the RT-qPCR positive serum was tested, which was expected to be early convalescent but for which the exact period between onset of disease and serum sampling was not known (17-07b). This serum sample did show some neutralizing potential at the 1:40 and 1:80 dilutions, but never reached the cut-off of $95 \%$ neutralization. However, the assay proved its worth for confirmation of SEOV infections as three sera in the clinical evaluation cohort could be serotyped as SEOV infections (Table 6 - 13-01b, 13-02c, and 16-15b). Interestingly, all three positive sera were convalescent, taken > 1 month after onset of disease. Additionally, all three sera showed relatively high IgG titers against the Murinae-borne orthohantaviruses in the diagnostic IFA (2- to 16-fold higher than serum 17-07b). For both PUUV and DOBV neutralizing antibodies could already be detected in the first few days after infection (Table 4 and (Horling et al., 1992)). These data, albeit limited, might suggest that neutralizing antibodies become detectable only relatively late after SEOV infection, implicating the need to test convalescent sera to confirm SEOV infections by MNT. However, solid evidence for these differences in kinetics can only be provided by extensive studies.

Lundkvist et al. described that acute and early convalescent human serum samples (taken $<1$ month post onset of disease) might be difficult to serotype due to high cross-reactivity in the VNT (Lundkvist et al., 1997). Here, relatively few problems with crossreactivity were encountered in both the validation and evaluation. Using 11 acute and 4 possibly early convalescent human sera, crossreactivity was only seen to a low extent and, if present, primarily within the serogroups. Only for one serum sample (Table 5 DOBV suspected 3), cross-neutralization toward the other serogroup was observed. This sample showed a relatively high neutralizing titer toward the infecting virus $\left(\mathrm{MNT}_{95}\right.$ of 640 , the highest neutralizing titer measured against DOBV in this study), which might explain the low levels of cross-neutralization toward PUUV and TULV. This explanation is supported by the notion that also $R$. norvegicus sera with high neutralizing titers against SEOV $(\geq 1280)$ showed some degree of cross-neutralization toward PUUV. Interestingly, however, cross-neutralization from the Arvicolinaeborne orthohantaviruses (PUUV and TULV) toward the Murinaeborne orthohantaviruses (DOBV and SEOV) was not observed during validation, even though some rodent and human sera had neutralizing titers of $\geq 5120$ toward either PUUV or TULV. It was, however, observed once in the diagnostic cohort (Table 6 - 17-09b) for which we have no clear explanation, other than the relatively early sampling date of the serum tested (17 days post onset of disease), which might have affected serotyping (Lundkvist et al., 1997).

The vast majority of cases (44/50) in our clinical cohort of orthohantavirus infections were identified as caused by PUUV, which confirms the assumption that PUUV is the major cause of orthohantavirus infections in Netherlands (Goeijenbier et al., 2014; Sane et al., 2014). Three human SEOV infections were confirmed of which one (Table 6 - 16-15) was previously described as the first proven SEOV case in Netherlands based on IFT serology and an epidemiological link to SEOV RNA positive rats (Swanink et al., 2018), and now confirmed by comparative VNT. Interestingly, the two other SEOV cases (Table 6- 13-01 and 13-02) confirmed in this study predated this case by almost three years, but were not recognized as SEOV cases using routine serological diagnostic assays (ELISA and IFA) at the time. This underlines the added 
TABLE 6 | MNT 95 titers for the diagnostic cohort of clinically suspected human orthohantavirus cases diagnosed with an orthohantavirus infection based on routine serology.

\begin{tabular}{|c|c|c|c|c|c|c|c|c|}
\hline \multirow{3}{*}{$\begin{array}{l}\text { Patient } \\
\text { ID }\end{array}$} & \multirow{3}{*}{$\begin{array}{c}\text { Serum collection (days post disease } \\
\text { onset) }\end{array}$} & \multirow{3}{*}{$\begin{array}{l}\text { Serogroup with highest reactivity in } \\
\text { IFA }\end{array}$} & \multicolumn{6}{|c|}{ Orthohantavirus $\mathrm{MNT}_{95}$} \\
\hline & & & \multicolumn{4}{|c|}{$95 \%$ neutralization titer } & \multirow{2}{*}{$\begin{array}{l}\text { Minimal Fold } \\
\text { change }\end{array}$} & \multirow[t]{2}{*}{ Conclusion } \\
\hline & & & PUUV & TULV & DOBV & SEOV & & \\
\hline $13-01 b$ & $>32$ & DOBV/HTNV/SAAV/SEOV & $<40$ & $<40$ & $<40$ & 160 & $\geq 8$ & SEOV \\
\hline $13-02 c$ & 75 & DOBV/HTNV/SAAV/SEOV & $<40$ & $<40$ & $<40$ & 160 & $\geq 8$ & SEOV \\
\hline $14-01$ & NA & PUUV/SNV & $\geq 5120$ & 40 & $<40$ & $<40$ & $\geq 128$ & PUUV \\
\hline $14-02$ & $<9$ & PUUV/SNV & 1,280 & 320 & $<40$ & $<40$ & 4 & PUUV \\
\hline $14-03$ & NA & PUUV/SNV & 2,560 & $<40$ & $<40$ & $<40$ & $\geq 128$ & PUUV \\
\hline $14-04 b$ & $>53$ & PUUV/SNV & 640 & 80 & $<40$ & $<40$ & 8 & PUUV \\
\hline $14-06$ & NA & PUUV/SNV & 2,560 & 40 & $<40$ & $<40$ & 64 & PUUV \\
\hline $14-07$ & NA & PUUV/SNV & 640 & $<40$ & $<40$ & $<40$ & $\geq 32$ & PUUV \\
\hline $15-01$ & NA & PUUV/SNV & $\geq 5,120$ & 80 & $<40$ & $<40$ & $\geq 64$ & PUUV \\
\hline $15-02$ & 7 & ND & 640 & 80 & $<40$ & $<40$ & 8 & PUUV \\
\hline $15-03$ & 10 & PUUV/SNV & 640 & 320 & $<40$ & $<40$ & 2 & Inconclusive \\
\hline $15-04$ & 6 & PUUV/SNV & 2,560 & $<40$ & $<40$ & $<40$ & $\geq 128$ & PUUV \\
\hline $15-05$ & 11 & ND & 640 & 80 & $<40$ & $<40$ & 8 & PUUV \\
\hline $15-06$ & NA & PUUV/SNV & 640 & $<40$ & $<40$ & $<40$ & $\geq 32$ & PUUV \\
\hline $15-07$ & NA & PUUV/SNV & $\geq 5,120$ & $<40$ & $<40$ & $<40$ & $>128$ & PUUV \\
\hline $16-01$ & $<3$ & PUUV/SNV & $\geq 5,120$ & 80 & $<40$ & $<40$ & $\geq 64$ & PUUV \\
\hline $16-02$ & 7 & ND & 1,280 & $<40$ & $<40$ & $<40$ & $\geq 64$ & PUUV \\
\hline $16-03$ & NA & PUUV/SNV & 640 & $<40$ & $<40$ & $<40$ & $\geq 32$ & PUUV \\
\hline $16-04$ & NA & PUUV/SNV & 2,560 & $<40$ & $<40$ & $<40$ & $\geq 128$ & PUUV \\
\hline $16-05$ & NA & PUUV/SNV & $\geq 5,120$ & 80 & $<40$ & $<40$ & $\geq 64$ & PUUV \\
\hline $16-06$ & NA & PUUV/SNV & 1,280 & $<40$ & $<40$ & $<40$ & $\geq 64$ & PUUV \\
\hline $16-08$ & NA & PUUV/SNV & 160 & $<40$ & $<40$ & $<40$ & $\geq 8$ & PUUV \\
\hline $16-09$ & NA & PUUV/SNV & 320 & $<40$ & $<40$ & $<40$ & $\geq 16$ & PUUV \\
\hline $16-10$ & 12 & PUUV/SNV & 640 & $<40$ & $<40$ & $<40$ & $\geq 32$ & PUUV \\
\hline $16-11$ & NA & PUUV/SNV & 640 & 40 & $<40$ & $<40$ & 16 & PUUV \\
\hline $16-12$ & NA & PUUV/SNV & 160 & 80 & $<40$ & $<40$ & 2 & Inconclusive \\
\hline $16-13$ & 0 & PUUV/SNV & $\geq 5,120$ & 40 & $<40$ & $<40$ & $\geq 128$ & PUUV \\
\hline $16-14$ & NA & PUUV/SNV & 320 & $<40$ & $<40$ & $<40$ & $\geq 16$ & PUUV \\
\hline $16-15 b$ & 30 & DOBV/HTNV/SAAV/SEOV & $<40$ & 40 & 40 & 160 & 4 & SEOV \\
\hline $16-16$ & NA & PUUV/SNV & 2,560 & 40 & $<40$ & $<40$ & 64 & PUUV \\
\hline $16-17$ & NA & PUUV/SNV & 1,280 & 40 & $<40$ & $<40$ & 32 & PUUV \\
\hline $16-18$ & NA & PUUV/SNV & 320 & $<40$ & $<40$ & $<40$ & $\geq 16$ & PUUV \\
\hline $17-01$ & 11 & ND & 640 & 160 & $<40$ & $<40$ & 4 & PUUV \\
\hline $17-03 b$ & 24 & PUUV/SNV & 640 & 40 & $<40$ & $<40$ & 8 & PUUV \\
\hline $17-04 b$ & NA & PUUV/SNV & 640 & $<40$ & $<40$ & $<40$ & $\geq 32$ & PUUV \\
\hline $17-05 b$ & 36 & PUUV/SNV & 640 & $<40$ & $<40$ & $<40$ & $\geq 32$ & PUUV \\
\hline $17-09 b$ & 17 & DOBV/HTNV/SAAV/SEOV & $<40$ & 80 & $<40$ & 40 & 2 & Inconclusive \\
\hline $17-10$ & NA & ND & 160 & $<40$ & $<40$ & $<40$ & $\geq 8$ & PUUV \\
\hline $17-11$ & NA & PUUV/SNV & 640 & $<40$ & $<40$ & $<40$ & $\geq 32$ & PUUV \\
\hline $17-12$ & NA & PUUV/SNV & 640 & $<40$ & $<40$ & $<40$ & $\geq 32$ & PUUV \\
\hline $17-15 b$ & 29 & PUUV/SNV & 1,280 & 40 & $<40$ & $<40$ & 32 & PUUV \\
\hline $17-16$ & 5 & ND & 2,560 & $<40$ & $<40$ & $<40$ & $\geq 128$ & PUUV \\
\hline $17-17$ & NA & PUUV/SNV & 2,560 & 40 & $<40$ & $<40$ & 64 & PUUV \\
\hline $17-18$ & NA & ND & 640 & $<40$ & $<40$ & $<40$ & $\geq 32$ & PUUV \\
\hline $17-19 b$ & 43 & PUUV/SNV & 2,560 & 160 & $<40$ & $<40$ & 16 & PUUV \\
\hline $17-20$ & 1 & PUUV/SNV & $\geq 5,120$ & $<40$ & $<40$ & $<40$ & $>128$ & PUUV \\
\hline $17-21$ & NA & PUUV/SNV & 1,280 & $<40$ & $<40$ & $<40$ & $\geq 64$ & PUUV \\
\hline $17-22$ & 8 & ND & 2,560 & $<40$ & $<40$ & $<40$ & $\geq 128$ & PUUV \\
\hline $17-24$ & NA & PUUV/SNV & 640 & 40 & $<40$ & $<40$ & 16 & PUUV \\
\hline $17-26$ & NA & ND & 1,280 & $<40$ & $<40$ & $<40$ & $\geq 64$ & PUUV \\
\hline
\end{tabular}

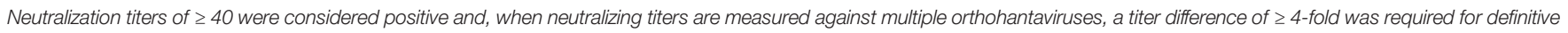
serotyping. The highest positive titer is highlighted in gray.

value of the use of comparative MNT for determination of causative orthohantavirus species. It must however be noted that evidence of human SEOV infections in Netherlands was already observed in the 1980s (Groen et al., 1991; Clement et al., 2019), however these cases were never confirmed by either RT-PCR or VNT.
No evidence for DOBV and TULV infections was found in the 50 patients included in our diagnostic cohort. DOBV infections are only known as imported cases (Geurts van Kessel et al., 2016). Its main reservoir (A. flavicollis) has only limited presence in Netherlands and is considered not to carry 
the virus. TULV, on the other hand, is circulating among the common vole populations at multiple locations in Netherlands (Reusken et al., 2008; Maas et al., 2017). However, no human TULV infections have been recognized in Netherlands so far, which is in line with the only sporadic human TULV cases reported in Europe to date (Schultze et al., 2002; Klempa et al., 2003; Zelena et al., 2013; Reynes et al., 2015), even though the virus is able to infect humans (Vapalahti et al., 1996; Mertens et al., 2011). To gain insight in the infection pressure of TULV in Netherlands dedicated seroprevalence studies are needed for which our developed MNT represents a crucial tool. Unfortunately, no human sera of the few TULV cases in Europe could be tested in the MNT. As sera from TULV-positive rodents could be clearly discerned from the other orthohantavirus-positive rodent sera using the comparative MNT, we expect that the assay is able to correctly serotype TULV-positive human sera as well. However, the exact performance of the MNT with human TULV sera remains to be tested.

In conclusion, we demonstrate the successful development and clinical implementation of a comparative European orthohantavirus MNT to determine the infecting virus species in European HFRS/NE patients. Identification of the causative species is needed for an adequate Public Health response, e.g. in the case of SEOV and pet rats, and can support individual patient care. The general absence of orthohantavirus neutralization tests in European reference laboratories (EVD-LabNet) illustrates that the implementation of virus neutralization tests for orthohantaviruses is not a straight forward procedure. We will address this issue by the roll-out of this test, including across laboratory performance comparisons, to multiple European laboratories (EVD-LabNet, pers. comm.) to support patient diagnostics, surveillance and Public Health responses.

\section{REFERENCES}

EVD-LabNet Directory search. Available at: https://www.evd-labnet.eu/evdlabnet-directory-search?genus=381-hantavirus (Accessed 15 December 2019).

Chu, Y. K., Jennings, G., Schmaljohn, A., Elgh, F., Hjelle, B., Lee, H. W., et al. (1995). Cross-neutralization of hantaviruses with immune sera from experimentally infected animals and from hemorrhagic fever with renal syndrome and hantavirus pulmonary syndrome patients. J. Infect. Dis. 172 (6), 1581-1584. doi: 10.1093/infdis/172.6.1581

Clement, J., Heyman, P., McKenna, P., Colson, P., and Avsic-Zupanc, T. (1997). The hantaviruses of Europe: from the bedside to the bench. Emerg. Infect. Dis. 3 (2), 205-211. doi: 10.3201/eid0302.970218

Clement, J., Frans, J., and Van Ranst, M. (2003). Human Tula virus infection or rat-bite fever? Eur. J. Clin. Microbiol. Infect. Dis. 22 (5), 332-333; author reply 334-335. doi: 10.1007/s10096-003-0921-7

Clement, J., Maes, P., and Van Ranst, M. (2014). Hemorrhagic Fever with Renal Syndrome in the New, and Hantavirus Pulmonary Syndrome in the Old World: paradi(se)gm lost or regained? Virus Res. 187, 55-58. doi: 10.1016/ j.virusres.2013.12.036

Clement, J., LeDuc, J. W., Lloyd, G., Reynes, J. M., McElhinney, L., Van Ranst, M., et al. (2019). Wild Rats, Laboratory Rats, Pet Rats: Global Seoul Hantavirus Disease Revisited. Viruses 11 (7). doi: 10.3390/v11070652

de Vries, A., Vennema, H., Bekker, D. L., Maas, M., Adema, J., Opsteegh, M., et al. (2016). Characterization of Puumala hantavirus in bank voles from two regions in the Netherlands where human cases occurred. J. Gen. Virol. 97 (7), 15001510. doi: 10.1099 /jgv.0.000481

Geurts van Kessel, C. H., Goeijenbier, M., Verner-Carlsson, J., Litjens, E., Bos, W. J., Pas, S. D., et al. (2016). Two clinical cases of renal syndrome caused by Dobrava/

\section{DATA AVAILABILITY STATEMENT}

The raw data supporting the conclusions of this article will be made available by the authors upon request, without undue reservation.

\section{ETHICS STATEMENT}

Ethical review and approval was not required for the study on human participants in accordance with the local legislation and institutional requirements. The patients/participants provided their written informed consent to participate in this study. The animal study was reviewed and approved by Dutch (DEC project numbers 200900164 and 201200208) and Slovenian Animal Ethics Committee (Administration for Food Safety, Veterinary Sector and Plant Protection: U34401-15/2018/8).

\section{AUTHOR CONTRIBUTIONS}

$\mathrm{TH}$, JR, and CR contributed to the design of the study. TH, IZ, $\mathrm{MH}$, and AV performed the experiments. JR, MM, TA- $\check{Z}, A V$, and $\mathrm{MK}$ provided critical samples for validation and assessment of the assay. TH and CR wrote the manuscript. All authors contributed to the article and approved the submitted version.

\section{SUPPLEMENTARY MATERIAL}

The Supplementary Material for this article can be found online at: https://www.frontiersin.org/articles/10.3389/fcimb.2020. 580478/full\#supplementary-material

Saaremaa hantaviruses imported to the Netherlands from Poland and Belaru2014. Infect. Ecol. Epidemiol. 6:30548. doi: 10.3402/iee.v6.30548

Goeijenbier, M., Hartskeerl, R. A., Reimerink, J., Verner-Carlsson, J., Wagenaar, J. F., Goris, M. G., et al. (2014). The hanta hunting study: underdiagnosis of Puumala hantavirus infections in symptomatic non-travelling leptospirosissuspected patients in the Netherlands, in 2010 and April to November 2011. Euro Surveill. 19 (32), pii=20878. doi: 10.2807/1560-7917.es2014.19.32.20878

Groen, J., Jordans, H. G., Clement, J. P., Rooijakkers, E. J., UytdeHaag, F. G., Dalrymple, J., et al. (1991). Identification of Hantavirus serotypes by testing of post-infection sera in immunofluorescence and enzyme-linked immunosorbent assays. J. Med. Virol. 33 (1), 26-32. doi: 10.1002/jmv.1890330106

Heider, H., Ziaja, B., Priemer, C., Lundkvist, A., Neyts, J., Kruger, D. H., et al. (2001). A chemiluminescence detection method of hantaviral antigens in neutralisation assays and inhibitor studies. J. Virol. Methods 96 (1), 17-23. doi: 10.1016/s0166-0934(01)00314-7

Hierholzer, J. C., and Killington, R. A. (1996). Virus Isolation and Quantitation (London, San Diego: Academic Press). doi: 10.1016/B978-012465330-6/50003-8

Hofmann, J., Meier, M., Enders, M., Fuhrer, A., Ettinger, J., Klempa, B., et al. (2014). Hantavirus disease in Germany due to infection with DobravaBelgrade virus genotype Kurkino. Clin. Microbiol. Infect. 20 (10), O648O655. doi: 10.1111/1469-0691.12543

Horling, J., Lundkvist, A., Huggins, J. W., and Niklasson, B. (1992). Antibodies to Puumala virus in humans determined by neutralization test. J. Virol. Methods 39 (1-2), 139-147. doi: 10.1016/0166-0934(92)90132-w

Kallio-Kokko, H., Laakkonen, J., Rizzoli, A., Tagliapietra, V., Cattadori, I., Perkins, S. E., et al. (2006). Hantavirus and arenavirus antibody prevalence in rodents and humans in Trentino, Northern Italy. Epidemiol. Infect. 134 (4), 830-836. doi: $10.1017 /$ s0950268805005431 
Klempa, B., Meisel, H., Rath, S., Bartel, J., Ulrich, R., and Kruger, D. H. (2003). Occurrence of renal and pulmonary syndrome in a region of northeast Germany where Tula hantavirus circulates. J. Clin. Microbiol. 41 (10), 48944897. doi: 10.1128/jcm.41.10.4894-4897.2003

Klempa, B., Avsic-Zupanc, T., Clement, J., Dzagurova, T. K., Henttonen, H., Heyman, P., et al. (2013). Complex evolution and epidemiology of DobravaBelgrade hantavirus: definition of genotypes and their characteristics. Arch. Virol. 158 (3), 521-529. doi: 10.1007/s00705-012-1514-5

Klimov, A., Balish, A., Veguilla, V., Sun, H., Schiffer, J., Lu, X., et al. (2012). Influenza virus titration, antigenic characterization, and serological methods for antibody detection. Methods Mol. Biol. 865, 25-51. doi: 10.1007/978-161779-621-0_3

Korva, M., Duh, D., Saksida, A., Trilar, T., and Avsic-Zupanc, T. (2009). The hantaviral load in tissues of naturally infected rodents. Microbes Infect. 11 (3), 344-351. doi: 10.1016/j.micinf.2008.12.016

Korva, M., Knap, N., Rus, K. R., Fajs, L., Grubelnik, G., Bremec, M., et al. (2013a). Phylogeographic diversity of pathogenic and non-pathogenic hantaviruses in slovenia. Viruses 5 (12), 3071-3087. doi: 10.3390/v5123071

Korva, M., Saksida, A., Kejzar, N., Schmaljohn, C., and Avsic-Zupanc, T. (2013b). Viral load and immune response dynamics in patients with haemorrhagic fever with renal syndrome. Clin. Microbiol. Infect. 19 (8), E358-E366. doi: 10.1111/ 1469-0691.12218

Kramski, M., Meisel, H., Klempa, B., Kruger, D. H., Pauli, G., and Nitsche, A. (2007). Detection and typing of human pathogenic hantaviruses by real-time reverse transcription-PCR and pyrosequencing. Clin. Chem. 53 (11), 18991905. doi: 10.1373/clinchem.2007.093245

Kruger, D. H., Schonrich, G., and Klempa, B. (2011). Human pathogenic hantaviruses and prevention of infection. Hum. Vaccin. 7 (6), 685-693. doi: 10.4161/hv.7.6.15197

Lederer, S., Lattwein, E., Hanke, M., Sonnenberg, K., Stoecker, W., Lundkvist, A., et al. (2013). Indirect immunofluorescence assay for the simultaneous detection of antibodies against clinically important old and new world hantaviruses. PloS Negl. Trop. Dis. 7 (4), e2157. doi: 10.1371/journal.pntd.0002157

Lee, P. W., Gibbs, C. J.Jr., Gajdusek, D. C., and Yanagihara, R. (1985). Serotypic classification of hantaviruses by indirect immunofluorescent antibody and plaque reduction neutralization tests. J. Clin. Microbiol. 22 (6), 940-944. doi: 10.1128/JCM.22.6.940-944.1985

Li, W., Cao, S., Zhang, Q., Li, J., Zhang, S., Wu, W., et al. (2017). Comparison of serological assays to titrate Hantaan and Seoul hantavirus-specific antibodies. Virol. J. 14 (1), 133. doi: 10.1186/s12985-017-0799-0

Lundkvist, A., Hukic, M., Horling, J., Gilljam, M., Nichol, S., and Niklasson, B. (1997). Puumala and Dobrava viruses cause hemorrhagic fever with renal syndrome in Bosnia-Herzegovina: evidence of highly cross-neutralizing antibody responses in early patient sera. J. Med. Virol. 53 (1), 51-59. doi: 10.1002/(SICI)1096-9071(199709)53:1<51::AID-JMV9>3.0.CO;2-P

Maas, M., de Vries, A., van Roon, A., Takumi, K., van der Giessen, J., and Rockx, B. (2017). High Prevalence of Tula Hantavirus in Common Voles in The Netherlands. Vector Borne Zoonotic Dis. 17 (3), 200-205. doi: 10.1089/vbz.2016.1995

Maas, M., De Vries, A., Reusken, C., Buijs, J., Goris, M., Hartskeerl, R., et al. (2018). Prevalence of Leptospira spp. and Seoul hantavirus in brown rats (Rattus norvegicus) in four regions in the Netherland-2015. Infect. Ecol. Epidemiol. 8 (1):1490135. doi: 10.1080/20008686.2018.1490135

Maes, P., Keyaerts, E., Li, S., Nlandu-Masunda, V., Clement, J., and Van Ranst, M. (2009). Replication reduction neutralization test, a quantitative RT-PCR-based technique for the detection of neutralizing hantavirus antibodies. J. Virol. Methods 159 (2), 295-299. doi: 10.1016/j.jviromet.2009.04.006

Mertens, M., Hofmann, J., Petraityte-Burneikiene, R., Ziller, M., Sasnauskas, K., Friedrich, R., et al. (2011). Seroprevalence study in forestry workers of a nonendemic region in eastern Germany reveals infections by Tula and DobravaBelgrade hantaviruses. Med. Microbiol. Immunol. 200 (4), 263-268. doi: 10.1007/s00430-011-0203-4

Nichol, S. T., Beaty, B. J., Elliot, R. M., Goldbach, R. W., Plyusnin, A., and Tesh, R. B. (2005). “The Bunyaviridae," in Virus Taxonomy: VIIIth Report of the International Committee on Taxonomy of Viruses. Eds. C. M. Fauquet, M. A. Mayo, J. Maniloff, U. Desselberger and L. A. Ball (London: Elsevier Academic Press), 695-716.

Niklasson, B., Jonsson, M., Lundkvist, A., Horling, J., and Tkachenko, E. (1991). Comparison of European isolates of viruses causing hemorrhagic fever with renal syndrome by a neutralization test. Am. J. Trop. Med. Hyg. 45 (6), 660-665. doi: 10.4269/ajtmh.1991.45.660

Padua, M., Souza, W. M., Lauretti, F., and Figueiredo, L. T. (2015). Development of a novel plaque reduction neutralisation test for hantavirus infection. Mem. Inst. Oswaldo Cruz 110 (5), 624-628. doi: 10.1590/0074-02760150102

Razzauti, M., Plyusnina, A., Sironen, T., Henttonen, H., and Plyusnin, A. (2009). Analysis of Puumala hantavirus in a bank vole population in northern Finland: evidence for co-circulation of two genetic lineages and frequent reassortment between strains. J. Gen. Virol. 90 (Pt 8), 1923-1931. doi: 10.1099/vir.0.011304-0

Reusken, C., and Heyman, P. (2013). Factors driving hantavirus emergence in Europe. Curr. Opin. Virol. 3 (1), 92-99. doi: 10.1016/j.coviro.2013.01.002

Reusken, C., de Vries, A., Adema, J., Vos, W., van der Giessen, J., Bekker, D., et al. (2008). First genetic detection of Tula hantavirus in wild rodents in the Netherlands. J. Infect. 57 (6), 500-503. doi: 10.1016/j.jinf.2008.09.032

Reuter, M., and Kruger, D. H. (2018). The nucleocapsid protein of hantaviruses: much more than a genome-wrapping protein. Virus Genes 54 (1), 5-16. doi: 10.1007/s11262-017-1522-3

Reynes, J. M., Carli, D., Boukezia, N., Debruyne, M., and Herti, S. (2015). Tula hantavirus infection in a hospitalised patient, France, June 2015. Euro Surveill. 20 (50), pii=30095. doi: 10.2807/1560-7917.Es.2015.20.50.30095

Sane, J., Reimerink, J., Harms, M., Bakker, J., Mughini-Gras, L., Schimmer, B., et al. (2014). Human hantavirus infections in the Netherlands. Emerg. Infect. Dis. 20 (12), 2107-2110. doi: 10.3201/eid2012.131886

Schultze, D., Lundkvist, A., Blauenstein, U., and Heyman, P. (2002). Tula virus infection associated with fever and exanthema after a wild rodent bite. Eur. J. Clin. Microbiol. Infect. Dis. 21 (4), 304-306. doi: 10.1007/s10096-002-0705-5

Swanink, C., Reimerink, J., Gisolf, J., de Vries, A., Claassen, M., Martens, L., et al. (2018). Autochthonous Human Case of Seoul Virus Infection, the Netherlands. Emerg. Infect. Dis. 24 (12), 2158-2163. doi: 10.3201/eid2412.180229

Takenaka, A., Gibbs, C. J.Jr., and Gajdusek, D. C. (1985). Antiviral neutralizing antibody to Hantaan virus as determined by plaque reduction technique. Arch. Virol. 84 (3-4), 197-206. doi: 10.1007/bf01378972

Tanishita, O., Takahashi, Y., Okuno, Y., Yamanishi, K., and Takahashi, M. (1984). Evaluation of focus reduction neutralization test with peroxidaseantiperoxidase staining technique for hemorrhagic fever with renal syndrome virus. J. Clin. Microbiol. 20 (6), 1213-1215. doi: 10.1128/ JCM.20.6.1213-1215.1984

Vaheri, A., Vapalahti, O., and Plyusnin, A. (2008). How to diagnose hantavirus infections and detect them in rodents and insectivores. Rev. Med. Virol. 18 (4), 277-288. doi: 10.1002/rmv.581

Vaheri, A., Strandin, T., Hepojoki, J., Sironen, T., Henttonen, H., Makela, S., et al. (2013). Uncovering the mysteries of hantavirus infections. Nat. Rev. Microbiol. 11 (8), 539-550. doi: 10.1038/nrmicro3066

Vapalahti, O., Lundkvist, A., Kukkonen, S. K., Cheng, Y., Gilljam, M., Kanerva, M., et al. (1996). Isolation and characterization of Tula virus, a distinct serotype in the genus Hantavirus, family Bunyaviridae. J. Gen. Virol. 77 ( Pt 12), 30633067. doi: 10.1099/0022-1317-77-12-3063

Vapalahti, O., Mustonen, J., Lundkvist, A., Henttonen, H., Plyusnin, A., and Vaheri, A. (2003). Hantavirus infections in Europe. Lancet Infect. Dis. 3 (10), 653-661. doi: 10.1016/s1473-3099(03)00774-6

Verner-Carlsson, J., Lohmus, M., Sundstrom, K., Strand, T. M., Verkerk, M., Reusken, C., et al. (2015). First evidence of Seoul hantavirus in the wild rat population in the Netherlands. Infect. Ecol. Epidemiol. 5:27215. doi: 10.3402/iee.v5.27215

Zelena, H., Mrazek, J., and Kuhn, T. (2013). Tula hantavirus infection in immunocompromised host, Czech Republic. Emerg. Infect. Dis. 19 (11), 1873-1875. doi: 10.3201/eid1911.130421

Conflict of Interest: The authors declare that the research was conducted in the absence of any commercial or financial relationships that could be construed as a potential conflict of interest.

Copyright (c) 2020 Hoornweg, Zutt, de Vries, Maas, Hoogerwerf, Avšič-Županc, Korva, Reimerink and Reusken. This is an open-access article distributed under the terms of the Creative Commons Attribution License (CC BY). The use, distribution or reproduction in other forums is permitted, provided the original author(s) and the copyright owner(s) are credited and that the original publication in this journal is cited, in accordance with accepted academic practice. No use, distribution or reproduction is permitted which does not comply with these terms. 